\title{
Quality of Life in Women With Urinary Tract Infections: Is Benign Disease a Misnomer?
}

\author{
Anne K. Ellis, MD, and Sarita Verma, LLB, MD
}

Background: The objective of this study was to undertake an exploratory evaluation of quality-of-life indicators for women suffering from urinary tract infections.

Metbods: The RAND 36-Item Health Survey 1.0 (SF-36) was administered to 47 women with a diagnosed urinary tract infection who were being cared for in the Family Medicine Center, Student Health Services, or Urology Outpatient Clinic. A control population of $\mathbf{7 1}$ women was obtained from the female members of an undergraduate geography class, a community basketball league, and a local women's choir.

Results: All subsections of the SF-36 quality-of-life indices were significantly decreased in the subject population compared with the control population (lower score indicates lower quality of life): patient general health perception $(63.3$ vs $78.9, P<.001)$ physical functioning $(76.6$ vs $87.6, P=.012)$, role limitation owing to physical health $(53.8$ vs $93.0, P<.001)$ and emotional health $(67.4$ vs $88.3, P<$ $.001)$, vitality (43.0 vs 64.9, $P<.001)$, emotional well-being $(64.4$ vs $80.2, P<.001)$, pain $(58.7$ vs 91.5, $P<.001)$, and social functioning $(60.4$ vs $90.4 . P<.001)$.

Conclusion: Suffering from an urinary tract infection has a detrimental influence on patient quality of life. The effect of urinary tract infections on women and their perception of quality of life have not been hitherto reported in the medical literature. The significant findings in this study call into question whether acute, non-life-threatening illness should be regarded as benign. (J Am Board Fam Pract 2000; 13:392-7.)

An estimated $10 \%$ to $20 \%$ of women have a urinary tract infection at some point in their lives. ${ }^{1}$ The condition is common, and urinary tract infections in women of childbearing age account for 6 to 7 million visits per year to family physicians in the United States. ${ }^{2}$ Current knowledge of the cause ${ }^{3}$ and risk factors, ${ }^{4,5}$ as well as the medical approach to diagnosis ${ }^{1}$ and management, ${ }^{6-8}$ of this condition is well-studied. Many studies have documented 3-day therapy and 7- or 10-day treatment with antibiotics to be equally effective. ${ }^{6}$ An investigation of the impact that urinary tract infections have on patients' quality of life while they are symptomatic is unprecedented. The topic is alluded to on occasion in the medical literature $e^{9,10}$ and in literature directed at the lay-person, ${ }^{11}$ but there have been no

Submitted, revised, 27 March 2000.

From the Department of Medicine (AKE), Kingston General Hospital, and the Department of Family Medicine (SV), Queen's University, Kingston, Ontario, Canada. Address reprint requests to Sarita Verma, LLB, MD, Department of Family Medicine, Queen's University, 220 Bagot St, Kingston, Ontario K7L 3G2. studies that attempt to quantify quality of life in urinary tract infections.

Quality of life is the subjective value that a person places on satisfaction with his or her life and is influenced by several factors. ${ }^{12}$ Several aspects of quality of life have been documented and defined as being measurable with consistent, reproducible findings. These aspects are physical functioning, role limitation caused by physical health, role limitation caused by mental health, vitality, emotional well-being, social functioning, pain, and patient perception of general health. These eight facets of quality of life are measured by the instrument chosen for this study, the SF-36 (RAND 36-Item Health Survey Version 1.0). This generic, healthrelated quality-of-life instrument was chosen for its brevity, its ease of scoring, and its well-documented validity ${ }^{13-16}$ and use. This instrument is not age, disease, or treatment specific. It can assess healthrelated quality-of-life outcomes, namely, those known to be most directly affected by disease and treatment. $^{14}$

While quality of life has not been extensively studied in acute illness, some evaluations using the 
SF-36 have validated its use during acute conditions. These evaluations have been limited to such acute illness experiences as seasonal allergic rhinitis and sports injuries.

Because an assessment of quality of life in women with urinary tract infections has not been addressed in the medical literature, we decided to begin with a descriptive pilot study. The primary objective was to use the SF-36 to test quality-of-life indices in women with urinary tract infections. Our goal was to compare quality-of-life scores of women suffering from urinary tract infections with those of reasonably age-matched control women (women of the same age-group who were not acutely ill). The secondary objective of this study was to determine whether changes in quality-of-life indices were correlated with the duration or severity of the urinary tract infection.

\section{Methods}

\section{Study Sites}

To ensure a diversity of patients recruited to become subjects for the study, three study sites were established. The Family Medicine Center has a patient population representative of a typical family practice, with a wide range of patient ages and conditions. Women who sought treatment for a urinary tract infection in this setting generally had experienced such an infection before. Student Health Services associated with Queen's University has a generally younger population base. Most patients who are cared for in this setting are between the ages of 18 and 30 years. To increase recruitment, the Urology Outpatient Clinic also recruited patients. All women from this study site had been referred by their primary care physician for treatment of recurrent urinary tract infections. These women also tended to be older ( 30 years old or older).

\section{Recruitment}

The on-site physician offered all participants with a diagnosed urinary tract infection an individual questionnaire to complete. The completed questionnaire provided demographic and pertinent medical information in addition to quality-of-life information using the SF-36. The standard form of this survey elicits information regarding the past 4 weeks of the participant's life. Because urinary tract infections are extremely acute conditions, the time frame was modified to reflect the past 24 hours. Also sought were the duration and severity (subjective assessment) of the urinary tract infection, and whether it was the participant's first urinary tract infection. Duration was defined as the time from first onset of symptoms to the time of seeking medical attention. The completion of the questionnaire was voluntary, confidential, and anonymous. Questionnaires were completed by the participants on their own, without prompting or aid by the investigator-physician.

\section{Inclusion and Exclusion Criteria}

Participants were included if they were female, were aged 18 to 64 years, provided informed consent to participate, and had their condition diagnosed by a physician based on a history of dysuria, frequency, and urgency as well as urinalysis results consistent with urinary tract infection. Supportive microscopy assisted this diagnosis but was not required. Positive cultures were not required because they would delay study inclusion.

Participants were excluded from the study if they were younger than 18 or older than 64 years, had conditions mimicking urinary tract infections (eg, yeast vaginitis, interstitial cystitis), were pregnant, or had anatomic abnormalities complicating their clinical picture (eg, vesicoureteral reflux disease).

\section{Control Participants}

A control group was established by offering an identical questionnaire to women aged 18 to 64 years who were members of the community not suffering from any acute medical illness (as determined by self-reporting on the questionnaire). Three major community groups were targeted for survey completion. A second-year undergraduate geography class was approached to match roughly by age the participants recruited through the Student Health Services. Also contacted was a community women's basketball league and a local women's choir, both of which contributed control participants from a wide age range who served to match the participants recruited thorough the Family Medicine Center and the Urology Outpatient Clinic. The participation of the control group was also voluntary and confidential. Otherwise healthy women were used as a control group. The other two options would have been to use participants as their own controls, or to use patients coming to the clinic for other reasons. The former 
option was not workable for logistic reasons because follow-up was so difficult to obtain, and with such a small study sample, retaining participants was a concern. With the latter option, there was the possibility that control participants might also be experiencing another acute illness, which could confound the results.

\section{Etbical Comstderations}

The protocol for this study was reviewed and approved by the Queen's University Health Sciences \& Affiliated Teaching Hospitals Research Ethics Board. Subject and control participants gave verbal consent to take part in the survey, documented by their completion of the questionnaire. Subject and control participants could withdraw consent at any time after commencement of the survey.

\section{Outcome Measures}

The primary endpoint for this study was to compare quality-of-life scores between control and subject populations for each of eight pertinent subsections of the SF-36. The subsection of health change was not analyzed, as it was not applicable to the context of the study.

Secondary endpoints included calculations of correlation between quality-of-life subsection scores and urinary tract infection duration and severity as reported by the participants.

\section{Statistical Metbods}

All questionnaires obtained from participants who met the necessary inclusion and exclusion criteria were analyzed as a data set.

Scores on the eight subsections of the SF-36 were compared between subject and control participant data using the Mann-Whitney test for nonparametric data. The equality of the mean ages between groups was tested using both the MannWhitney and the independent samples $t$ test. Spearman correlation coefficient was used to assess the correlation between quality-of-life subsets and urinary tract infection severity and duration, as these data were nonparametric. The prevalence of comorbid conditions was compared using the chisquare test and the Fisher exact test where applicable. Statistical analyses were conducted using SPSS software. ${ }^{17}$

\section{Results}

\section{Subject Population}

Forty-nine women volunteered to complete the study questionnaire. Of these women, 2 did not meet the inclusion criteria based on age, leaving a study sample of 47 patients. Of these patients, 22 were from the Student Health Services population, 18 were from the Family Medicine Center, and 7 were from the Urology Outpatient Clinic.

The mean age of subject population was 32.3 years with a standard deviation of 12.5 years. Of those who completed the questions on urinary tract infection duration and severity, the average severity rating was 2.2 on a scale of 1 to 4 (mild $=1$, moderate $=2$, severe $=3$, very severe $=4$ ), and the average duration of urinary tract infections was 4.2 days, with a standard deviation of 3.3 days. It is of note, however, that only $74.5 \%$ of participants completed the duration question, and $83.0 \%$ answered the severity question.

\section{Control Population}

The questionnaire was offered to a total of 77 women to be completed on a voluntary basis. Two women refused to participate, and 4 were excluded based on age criteria or the presence of an acute medical condition. Thus, a control population of 71 women was established. Forty-five were from a local university geography class, 26 were from a community choir group, and 6 were from a local sports league.

The average age of the women in the control population was 34.0 years with a standard deviation of 12.8 years.

\section{Missing Data}

Sections of the questionnaires not completed by subject and control participants were minimal and were limited to two questionnaires with one missed question each. Mean scores were adjusted appropriately for those individual sections, accounting for the resultant change in the denominator.

\section{Comparison of Groups}

The primary objective of this study was to compare quality-of-life indices in women suffering from urinary tract infections with those of reasonably agematched controls (women of the same age-group who were not acutely ill). The mean ages of the 
Table 1. Comorbid Conditions of Subject and Control Populations.

\begin{tabular}{lccc}
\hline & $\begin{array}{c}\text { Subject } \\
\text { No. } \\
\text { Comorbidities }\end{array}$ & $\begin{array}{c}\text { Control } \\
\text { No. } \\
(\%)\end{array}$ & $P$ Value \\
\hline All conditions & $18(38.3)$ & $26(35.2)$ & .854 \\
Gastrointestinal & $2(4.3)$ & $5(7.0)$ & .701 \\
Genitourinary & $2(4.3)$ & $0(0.0)$ & .157 \\
Asthma and allergies & $6(12.8)$ & $9(12.7)$ & .989 \\
Metabolic & $2(4.3)$ & $2(2.8)$ & .673 \\
Musculoskeletal & $2(4.3)$ & $4(5.6)$ & .860 \\
Psychiatric & $5(10.6)$ & $5(7.0)$ & .492 \\
Hypertension & $5(10.6)$ & $2(2.8)$ & .078 \\
Miscellaneous & $1(2.1)$ & $3(4.2)$ & .538 \\
\hline
\end{tabular}

subject and control populations were statistically similar $(P=.646)$.

All eight subsections of the SF-36 indicated reduced quality of life in women suffering from a urinary tract infection when compared with control participants. Although the control group was not specifically matched for comorbid conditions, the control population contained a comparable proportion of these women (Table 1). The proportions of certain disease categories were all statistically similar. Only the condition of hypertension approached a level of significant difference at $P=$ .078 . As one can observe from Table 1, however, the number of patients involved in this category is not a large percentage of overall participants.

Even though all subsections were shown to be significantly different, certain subsections showed larger deficits in quality of life than others. Particularly affected by the presence of a urinary tract infection were the areas of role limitation because of physical health, pain, and social function (Figure 1).
The secondary objective of this study was to determine whether changes in quality-of-life indices were correlated with urinary tract infection duration or severity. Duration of urinary tract infection did not appear to have an important impact on quality of life. The only significant correlation between duration and any of the SF-36 subsections was in role limitation caused by mental health (rho $=-0.476, P=.004)$. Severity of urinary tract infection, on the other hand, was significantly correlated with several of the SF-36 subsection scores, including vitality (rho $=-0.354, P=.027$ ), pain (rho $=-0.452, P=.004)$ and role limitation caused by physical health (rho $=-0.346, P=$ .033 ), with possible correlation with social function (rho $=-0.297, P=.067)$.

\section{Discussion}

Quality of life has, of late, achieved respectability both as a matter of clinical concern and as a focus of research. Its use is spreading, and its importance growing. ${ }^{18}$ It has become an expected measure of success in many clinical trials, a criterion for licensing new medicines in some jurisdictions, ${ }^{19}$ and the focus of a specific journal (Quality of Life Research, published by Rapid Communications, Oxford, England).

This pilot study of quality of life in women with urinary tract infections involved a relatively small sample size, but despite this limitation, statistically significant results have been shown. This finding suggests that a larger scale study of this research question might be not only warranted but also extrapolated to other acute illnesses previously thought to be relatively benign.

\section{SF-36 Physical Parameters}

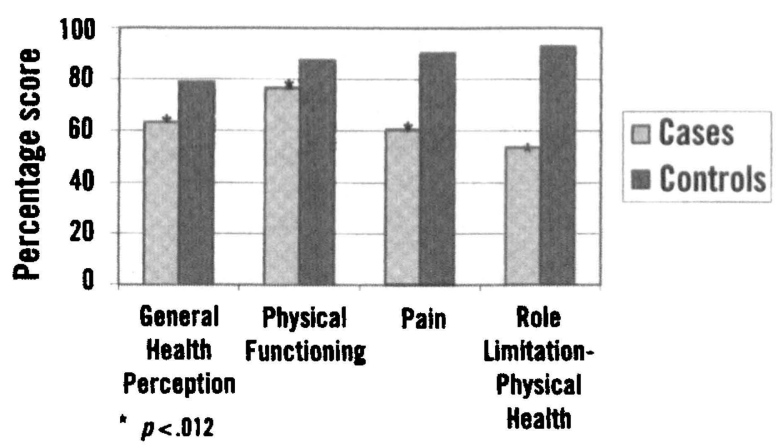

SF-36 Mental Parameters

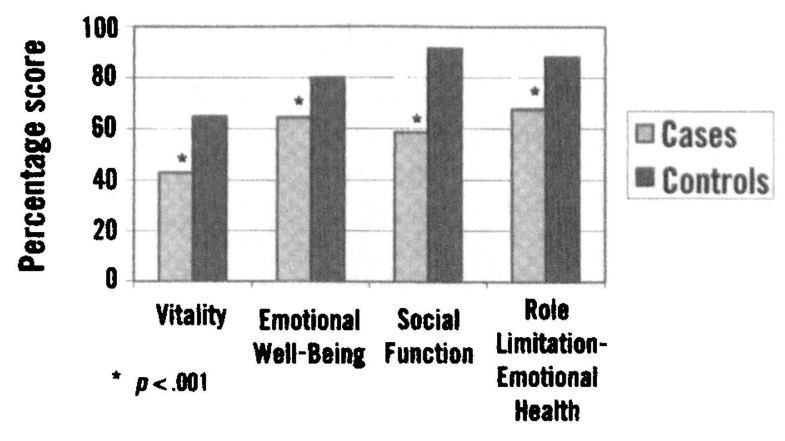

Figure 1. Mean scores of The RAND 36-Item Health Survey 1.0 (SF-36) subsections. 
Most trials assessing quality of life focus on chronic illnesses, and with just cause. The acute illness experience, however, is an equally valid target for research. It is important to determine the optimal management for all of our patient's needs, because an illness, although short-lived and not life-threatening, could have an important impact on the patients daily activities, social function, and emotional well-being. The results of this study call into question the presumptive benign nature of urinary tract infections and describe the impact of the disease from the patient's perspective of the illness experience. Not only was a statistically significant impact on quality of life detected in a small study population, but it was achieved using a generic instrument, which represents a documented compromise in measurement precision. ${ }^{20}$

The primary objective of this study was to determine and quantify the differences in quality of life between women suffering from urinary tract infections and women of equal age who were not acutely ill. All eight parameters of the SF-36 were significantly lower for women with urinary tract infections than for their healthy counterparts. This finding suggests that the symptoms experienced by women during a urinary tract infection (frequency, urgency, dysuria) affect all aspects of their quality of life (including vitality and emotional well-being).

Interestingly, urinary tract infection strongly influenced the parameters of role limitation caused by physical health, pain, and social function, especially the women's ability to fulfill their various life roles. In fact, the difference between subject participants and control participants in this subsection was even greater than the difference between pain scores, indicating that limitations on role fulfillment are brought about by other symptoms as well (ie, frequency, urgency). The difference in social function scores of women with urinary tract infections compared with control women was almost as great as the difference in pain scores. It would appear, therefore, that the symptoms of a urinary tract infection are more than simply discomfort and an inconvenience.

The secondary objective of the study was to determine whether the impact on quality of life was related to disease severity or duration. The results of these analyses were somewhat difficult to interpret in light of the finding that some subsections of the quality-of-life parameters examined correlated with urinary tract infection duration and severity, and others did not. It seems reasonable to conclude that urinary tract infection severity is partially correlated with quality of life, as more than one half of the subsections showed a significant correlation.

Limitations of this study include the small sample size. As this study was to be a preliminary investigation in this field, however, we believed that a smaller sample size would not seriously detract from determining whether further investigation was warranted. Given that the participants reported statistical differences in all sections of the SF-36, most with significance at the $P<.001$ level, it would seem that the small sample size did not interfere with the ability to pick up differences between subject and control participants.

Another concern was that the women coming to a specialist for treatment would have an increased deficiency in quality of life, and their inclusion in the data set would lead to an artificial statistical significance to the scores. To examine this possibility, the data set was again analyzed, omitting the data of the women from the urology clinic. Statistically significant differences were maintained in all subsections except for physical functioning $(P=$ $.105)$. Thus, the impact on quality of life for women with urinary tract infections is significant regardless of treatment setting.

Also considered in this study was the possible difference between subject and control participants in the proportion with the comorbid condition of hypertension. Even though the chi-square analysis showed nonsignificant differences, the $P$ value was .078 , suggesting that a difference could still be possible. Previous studies have documented decreased quality of life as measured by the SF-36 in patients with hypertension, ${ }^{21}$ thus it could be argued that some of the differences detected between subject and control populations were due to the higher proportion of patients with hypertension in the subject population. On further analysis of the data set with the Fisher exact test, which is more accurate for smaller numbers (specifically when the number of cases is equal to or less than 5), the difference was definitely nonsignificant $(P=$ .1132). Thus, the presence of more patients with hypertension is unlikely to be a factor.

A final possible limitation of this study was the change of the time frame of the acute version SF-36 from 1 week to 24 hours. This change has not been formally validated and might therefore represent a complicating factor. 


\section{Conclusions}

Suffering from a urinary tract infection significantly affected many aspects of quality of life of the women who completed our survey questionnaire. Quality of life is a well-recognized and valid portion of a patient's overall assessment, and a woman's symptoms should be taken seriously as a disease process with an important associated illness experience, albeit one of short duration. Given that the effect on quality of life is so far-reaching, involving social function, vitality, and emotional well-being, physicians might consider modifying their management of urinary tract infection to introduce preventative strategies earlier. Self-treatment and postcoital prophylaxis regimens have met with particular success. ${ }^{4,7}$ Physicians might also consider adding a urinary tract analgesic (phenazopyridine) to the treatment of symptoms for those patients with significant pain. The results of this study suggest that further investigation into the impact on quality of life on this and other acute so-called benign conditions is warranted.

The authors wish to acknowledge the physicians and staff of the Family Medicine Center, Student Health Services, and Urology Outpatients Clinics in Kingston, Ontario, for their role in patient recruitment. Additionally, Dr. Mark Lundie, Kingston General Hospital, provided an assistive role in the statistical analysis.

\section{References}

1. Johnson JR, Stamm WE. Urinary tract infections in women: diagnosis and treatment. Ann Intern Med 1989;111:906-17.

2. Stamm WE, Hooton TM, Johnson JR, et al. Urinary tract infections: from pathogenesis to treatment. J Infect Dis 1989;159:400-6.

3. Johnson JR, Stamm WE. Diagnosis and treatment of acute urinary tract infections. Infect Dis Clin North Am 1987;1:773-91.

4. Remis RS, Gurwith MJ, Gurwith D, Hargrett-Bean NT, Layde PM. Risk factors for urinary tract infection. Am J Epidemiol 1987;126:685-94.

5. Foxman B, Chi JW. Health behavior and urinary tract infection in college-aged women. J Clin Epidemiol 1990;43:329-37.

6. Stamm WE, Hooton TM. Management of urinary tract infections in adults. N Engl J Med 1993;329: 1328-34.

7. Elder NC. Acute urinary tract infection in women.
What kind of antibiotic therapy is optimal? Postgrad Med 1992;92:159-62, 165-6, 172.

8. Johnson MA. Urinary tract infections in women. Am Fam Physician 1990;41:565-71.

9. Wilkie ME, Almond MK, Marsh FP. Diagnosis and management of urinary tract infection in adults. BMJ 1992;305:1137-41.

10. Wise BG, Cardozo LD. Urinary urgency in women. Br J Hosp Med 1993;50:243-50.

11. Cystitis and urethritis. In: Chalker R, Whitmore KE. Overcoming bladder disorders: compassionate, authoritative, medical and self-help solutions for incontinence, cystitis, interstitial cystitis, prostate problems, bladder cancer. New York: HarperTrade, 1990:99.

12. Sullivan MB, Sullivan LG, Kral JG. Quality of life assessment in obesity: physical, psychological, and social function. Gastroenterol Clin North Am 1987; 16:433-42.

13. Brazier JE, Harper R, Jones NM, et al. Validating the SF-36 health survey questionnaire: new outcome measure for primary care. BMJ 1992;305:160-4.

14. Stewart AL, Hays RD, Ware JE Jr. The MOS shortform general health survey. Reliability and validity in a patient population. Med Care 1988;26:724-35.

15. McHorney CA, Ware JE Jr, Raczek AE. The MOS 36-item short form health survey (SF-36): II. Psychometric and clinical tests of validity in measuring physical and mental health constructs. Med Care 1993;31:247-63.

16. McHorney CA, Ware JE Jr, Rogers WH, Raczek A, $\mathrm{Lu} \mathrm{JF}$. The validity and relative precision of MOS short and long form health status scales and Dartmouth COOP charts: Results from the medical outcomes study. Med Care 1992;30:MS253-65.

17. SPSS version 7.0 for Windows [computer program] Chicago: SPSS, 1998.

18. Spilker B. Introduction. In: Spilker B, editor. Quality of life and pharmacoeconomics in clinical trials, ed 2. Philadelphia: Lippincott-Raven Publishers, 1996:110.

19. Miller L, Vestal R, Dalton M, Atwood B, Perkins JG, Lyon G. Quality of life II. Oncology: regulatory/ scientific aspects and the drug approval process. J Clin Res Pharmacoepidemiol 1990;4:39-53.

20. Ware JE. The SF-36 Health Survey. In: Spilker B, editor. Quality of life and pharmacoeconomics in clinical trials, ed 2. Philadelphia: Lippincott-Raven Publishers, 1996:337-345.

21. Kusek JW, Lee JY, Smith DE, et al. Effect of blood pressure control and antihypertensive drug regimen on quality of life: The African American study of kidney disease and hypertension (AASK) pilot study. Control Clin Trial 1996;16:40S-46S. 